\title{
ON PHASE TRANSITIONS FOR $P$-ADIC POTTS MODEL WITH COMPETING INTERACTIONS ON A CAYLEY TREE
}

\author{
F.M. MUKHAMEDOV, U.A. ROZIKOV, AND J.F.F. MENDES
}

\begin{abstract}
In the paper we considere three state $p$-adic Potts model with competing interactions on a Cayley tree of order two. We reduce a problem of describing of the $p$-adic Gibbs measures to the solution of certain recursive equation, and using it we will prove that a phase transition occurs if and only if $p=3$ for any value (non zero) of interactions. As well, we completely solve the uniqueness problem for the considered model in a $p$-adic context. Namely, if $p \neq 3$ then there is only a unique Gibbs measure the model.
\end{abstract}

Mathematics Subject Classification: 46S10, 82B26, 12 J12.

Key words: $p$-adic field, Potts model, Cayley tree, Gibbs measure, phase transition, uniqueness.

\section{INTRODUCTION}

A non-Kolmogorovian probability models [7],[8] in that probabilities belong to the filed of $p$-adic numbers $\mathbb{Q}_{p}$ were developed in connection with $p$-adic quantum models (see for example, [1],[6],[14],[22]). In [7],[9] a measure-theoretical axiomatics of the $p$-adic probability theory were proceeded. In [10],[12] certain, various limit theorems for $p$-adic valued probabilities have been proved. In [11] the theory of stochastic processes with values in $p$-adic and more general non-Archimedean fields having probability distributions with non-Archimedean values has been developed. There a non-Archimedean analogue of the Kolmogorov theorem was proved, that gives the opportunity to construct wide classes of stochastic processes by using finite dimensional probability distributions. This allowed us to begin the study and the development of certain problems of statistical mechanics in a context of the $p$-adic probability theory. In [16],[17] we have developed the $p$-adic probability theory approaches to study some models with nearest neighbor interactions on the Cayley tree, such as Ising and Potts models ${ }^{1}$. In those papers we investigated the set of $p$-adic Gibbs measures and a problem of phase transitions . Note that the $p$-adic Gibbs measures, associated with those models, enable Markov property.

\footnotetext{
${ }^{1}$ The classical (real value) contra parts of such models were considered in [3], [5]
} 
In the present paper we consider three state $p$-adic Potts models with competing interactions on a Cayley tree order two. We note that the models on a Cayley tree with competing interactions have been studied extensively (see Refs. [15],[20]) since the appearance of the Vannimenus model (see Ref.[21]), in which the physical motivations for the urgency of study such models was presented. In all of these works no exact solutions of the phase transition problem were found, but some solutions for specific parameter values were presented. In this paper we can completely solve the uniqueness problem for the considered model in a $p$-adic context. Basically, in the real case there are only sufficient conditions (like Dobrushin condition [4]) for the uniqueness of a Gibbs measure of certain models. We show that for the model under consideration there is a phase transition if and only if $p=3$ as well.

\section{PRELIMINARIES}

2.1. $p$-adic numbers and measures. Let $\mathbb{Q}$ be the field of rational numbers. Throughout the paper $p$ will be a fixed prime number. Every rational number $x \neq 0$ can be represented in the form $x=p^{r} \frac{n}{m}$, where $r, n \in \mathbb{Z}, m$ is a positive integer and $p, n, m$ are relatively prime. The $p$-adic norm of $x$ is given by $|x|_{p}=p^{-r}$ and $|0|_{p}=0$. This norm satisfies so called the strong triangle inequality

$$
|x+y|_{p} \leq \max \left\{|x|_{p},|y|_{p}\right\} .
$$

This is a ultrametricity of the norm. The completion of $\mathbb{Q}$ with respect to $p$-adic norm defines the $p$-adic field which is denoted by $\mathbb{Q}_{p}$. Let $B(a, r)=$ $\left\{x \in \mathbb{Q}_{p}|| x-\left.a\right|_{p}<r\right\}$, where $a \in \mathbb{Q}_{p}, r>0$. By $\log _{p}$ and $\exp _{p}$ we mean $p$-adic logarithm and exponential which are defined as series with the usual way (see, for more details [13]). The domain of converge for them are $B(1,1)$ and $B\left(0, p^{-1 /(p-1)}\right)$ respectively. These two functions have the following properties (see $[13,22])$ :

$\left|\exp _{p}(x)\right|_{p}=1, \quad\left|\exp _{p}(x)-1\right|_{p}=|x|_{p}<1, \quad\left|\log _{p}(1+x)\right|_{p}=|x|_{p}<p^{-1 /(p-1)}$

and

$$
\log _{p}\left(\exp _{p}(x)\right)=x, \exp _{p}\left(\log _{p}(1+x)\right)=1+x .
$$

Let $(X, \mathcal{B})$ be a space, where $\mathcal{B}$ is an algebra of subsets $X$. A function $\mu: \mathcal{B} \rightarrow \mathbb{Q}_{p}$ is said to be a $p$-adic measure if for any $A_{1}, \ldots, A_{n} \subset \mathcal{B}$ such that $A_{i} \cap A_{j}=\emptyset(i \neq j)$

$$
\mu\left(\bigcup_{j=1}^{n} A_{j}\right)=\sum_{j=1}^{n} \mu\left(A_{j}\right) .
$$


A $p$-adic measure is called a probability measure if $\mu(X)=1$. A $p$-adic probability measure $\mu$ is called bounded if $\sup \left\{|\mu(A)|_{p} \mid A \in \mathcal{B}\right\}<\infty$.

For more detail information about $p$-adic measures we refer to $[7,9]$.

2.2. The Cayley tree. The Cayley tree $\Gamma^{k}$ of order $k \geq 1$ is an infinite tree, i.e., a graph without cycles, such that each vertex of which lies on $k+1$ edges . Let $\Gamma^{k}=(V, \Lambda)$, where $V$ is the set of vertices of $\Gamma^{k}, \Lambda$ is the set of edges of $\Gamma^{k}$. The vertices $x$ and $y$ are called nearest neighbor, which is denoted by $l=\langle x, y\rangle$ if there exists an edge connecting them. A collection of the pairs $\left.\left\langle x, x_{1}\right\rangle, \ldots,<x_{d-1}, y\right\rangle$ is called $a$ path from $x$ to $y$. The distance $d(x, y), x, y \in V$ is the length of the shortest path from $x$ to $y$ in $\mathrm{V}$.

For the fixed $x^{0} \in V$ we set

$$
\begin{gathered}
W_{n}=\left\{x \in V \mid d\left(x, x^{0}\right)=n\right\}, \quad V_{n}=\bigcup_{m=1}^{n} W_{m}, \\
L_{n}=\left\{l=<x, y>\in L \mid x, y \in V_{n}\right\},
\end{gathered}
$$

for a fixed point $x^{0} \in V$.

Denote

$$
S(x)=\left\{y \in W_{n+1} \mid d(x, y)=1\right\}, x \in W_{n} .
$$

The defined set is called the set of direct successors. Observe that any vertex $x \neq x^{0}$ has $k$ direct successors and $x^{0}$ has $k+1$.

Two vertices $x, y \in V$ is called one level next-nearest-neighboring vertices if there is a vertex $z \in V$ such that $x, y \in S(z)$ and they are denoted by $>x, y<$. In this case the vertices $x, z, y$ are called ternary and denoted by $\langle x, z, y>$.

In the sequel we will consider semi-infinite Cayley tree $J^{2}$ of order 2, i.e. an infinite graph without cycles with 3 edges issuing from each vertex except for $x^{0}$ and with 2 edges issuing from the vertex $x^{0}$.

2.3. The model. Let $\mathbb{Q}_{p}$ be the field of $p$-adic numbers. By $\mathbb{Q}_{p}^{q-1}$ we denote $\underbrace{\mathbb{Q}_{p} \times \ldots \times \mathbb{Q}_{p}}_{q-1}$. The norm $\|x\|_{p}$ of an element $x \in \mathbb{Q}_{p}^{q-1}$ is defined by $\|x\|_{p}=\max _{1 \leq i \leq q-1}\left\{\left|x_{i}\right|_{p}\right\}$, here $x=\left(x_{1}, \ldots, x_{q-1}\right)$. By $x y$ we mean the bilinear form on $\bar{Q}_{p}^{q-1}$ defined by

$$
x y=\sum_{i=1}^{q-1} x_{i} y_{i}, \quad x=\left(x_{1}, \cdots, x_{q-1}\right), y=\left(y_{1}, \cdots, y_{q-1}\right) .
$$


Let $\Psi=\left\{\zeta_{1}, \zeta_{2}, \ldots, \zeta_{q}\right\}$, where $\zeta_{1}, \zeta_{2}, \ldots, \zeta_{q}$ are elements of $\mathbb{Q}_{p}^{q-1}$ such that $\left\|\zeta_{i}\right\|_{p}=1, i=1,2, \ldots, q$ and

$$
\zeta_{i} \zeta_{j}=\left\{\begin{array}{l}
1, \text { for } i=j, \\
0, \text { for } i \neq j
\end{array} \quad(i, j=1,2, \ldots, q-1), \zeta_{q}=\sum_{i=1}^{q-1} \zeta_{i}\right.
$$

Let $h \in \mathbb{Q}_{p}^{q-1}$, then we have $h=\sum_{i=1}^{q-1} h_{i} \zeta_{i}$ and

$$
h \zeta_{i}=\left\{\begin{array}{l}
h_{i}, \text { for } i=1,2, \ldots, q-1, \\
\sum_{i=1}^{q-1} h_{i}, \text { for } i=q
\end{array}\right.
$$

We consider the $p$-adic Potts model where spin takes values in the set $\Psi$ and is assigned to the vertices of the tree $J^{2}=(V, \Lambda)$. A configuration $\sigma$ on $V$ is then defined as a function $x \in V \rightarrow \sigma(x) \in \Psi$; in a similar fashion one defines a configuration $\sigma_{n}$ and $\sigma^{(n)}$ on $V_{n}$ and $W_{n}$ respectively. The set of all configurations on $V$ (resp. $V_{n}, W_{n}$ ) coincides with $\Omega=\Psi^{V}$ (resp. $\left.\Omega_{V_{n}}=\Psi^{V_{n}}, \quad \Omega_{W_{n}}=\Psi^{W_{n}}\right)$. One can see that $\Omega_{V_{n}}=\Omega_{V_{n-1}} \times \Omega_{W_{n}}$. Using this, for given configurations $\sigma_{n-1} \in \Omega_{V_{n-1}}$ and $\sigma^{(n)} \in \Omega_{W_{n}}$ we define their concatenations by

$$
\sigma_{n-1} \vee \sigma^{(n)}=\left\{\left\{\sigma_{n}(x), x \in V_{n-1}\right\},\left\{\sigma^{(n)}(y), y \in W_{n}\right\}\right\} .
$$

It is clear that $\sigma_{n-1} \vee \sigma^{(n)} \in \Omega_{V_{n}}$.

The Hamiltonian $H_{n}: \Omega_{V_{n}} \rightarrow \mathbb{Q}_{p}$ of the $p$-adic Potts model with competing interactions has the form

$$
\begin{aligned}
H_{n}(\sigma)= & -\sum_{<x, y>\in L_{n}} J_{x, y} \delta_{\sigma(x), \sigma(y)}-\sum_{>x, y<: x, y \in V_{n}} K_{x, y} \delta_{\sigma(x), \sigma(y)} \\
& -H \sum_{x \in V_{n}} \delta_{\zeta_{3}, \sigma(x)}, \quad n \in \mathbb{N}
\end{aligned}
$$

here $\sigma \in \Omega_{V_{n}}, \delta$ is the Kronecker symbol and

$$
\left\{\begin{array}{l}
\left|J_{x, y}\right|_{p}<p^{-1 /(p-1)}, \quad \forall<x, y> \\
\left|K_{u, v}\right|_{p}<p^{-1 /(p-1)}, \quad \forall>u, v< \\
|H|_{p}<p^{-1 /(p-1)}
\end{array}\right.
$$

\section{EXISTENCE OF PHASE TRANSITION}

In this section we give a construction of Gibbs measures for the three state $(q=3) p$-adic Potts model with competing interactions on a semi-infinite Cayley tree $J^{2}$ of order 2, and establish a phase transition for it.

In the sequel we will assume that the condition (2.5) is satisfied. Let $\mathbf{h}$ : $x \in V \rightarrow h_{x} \in \mathbb{Q}_{p}^{q-1}$ be a function of $x \in V$ such that $\left\|h_{x}\right\|_{p}<p^{-1 /(p-1)}$ 
for all $x \in V$. Given $n=1,2, \ldots$ consider a $p$-adic probability measure $\mu_{\mathbf{h}}^{(n)}$ on $\Omega_{V_{n}}$ defined by

$$
\mu_{\mathbf{h}}^{(n)}(\sigma)=Z_{n}^{-1} \exp _{p}\left\{-H_{n}(\sigma)+\sum_{x \in W_{n}} h_{x} \sigma(x)\right\}
$$

Here, as before, $\sigma \in \Omega_{V_{n}}$ and $Z_{n}$ is the corresponding partition function:

$$
Z_{n}=\sum_{\tilde{\sigma} \in \Omega_{V_{n}}} \exp _{p}\left\{-H(\tilde{\sigma})+\sum_{x \in W_{n}} h_{x} \tilde{\sigma}(x)\right\} .
$$

Note that the measures $\mu_{\mathbf{h}}^{(n)}$ are well defined, since from (2.5), $\left\|h_{x}\right\|_{p}<$ $p^{-1 /(p-1)}$ and the strong triangle inequality one gets

$$
\left|H_{n}(\sigma)+\sum_{x \in W_{n}} h_{x} \sigma(x)\right|_{p}<p^{-1 /(p-1)}
$$

for any $n \in \mathbb{N}$, which enables the existence of the measures (3.1). The compatibility condition for $\mu_{\mathbf{h}}^{(n)}, n \geq 1$ is given by the equality

$$
\sum_{\sigma^{(n)} \in \Omega_{W_{n}}} \mu_{\mathbf{h}}^{(n)}\left(\sigma_{n-1} \vee \sigma^{(n)}\right)=\mu_{\mathbf{h}}^{(n-1)}\left(\sigma_{n-1}\right) .
$$

We note that an analog of the Kolmogorov extension theorem for distributions can be proved for the $p$-adic measures given by (3.1) (see [11]). Then according to the Kolmogorov theorem there exists a unique $p$-adic measure $\mu_{\mathbf{h}}$ on $\Omega$ such that for every $n=1,2, \ldots$ and $\sigma_{n} \in \Omega_{n}$ the equality holds

$$
\mu_{\mathbf{h}}\left(\left\{\left.\sigma\right|_{V_{n}}=\sigma_{n}\right\}\right)=\mu_{\mathbf{h}}^{(n)}\left(\sigma_{n}\right),
$$

which will be called a $p$-adic Gibbs measure for the considered model. It is clear that the measure $\mu_{\mathbf{h}}$ depends on the function $\mathbf{h}$. By $\mathcal{S}$ we denote the set of all $p$-adic Gibbs measures associated with functions $\mathbf{h}=\left(h_{x}, x \in V\right)$. If $|\mathcal{S}| \geq 2$, then we say that for this model there exists a phase transition, otherwise, we say there is no phase transition ( here $|A|$ means the cardinality of a set $A$ ). In other words, the phase transition means that there are two different functions $\mathbf{h}=\left(h_{x}, x \in V\right)$ and $\mathbf{s}=\left(s_{x}, x \in V\right)$ for which there exist two $\mu_{\mathbf{h}}$ and $\mu_{\mathbf{s}} p$-adic Gibbs measures on $\Omega$, respectively.

Using (3.1) and the argument of the proof of Theorem 3.2 [16] we may obtain that the measures $\mu_{\mathbf{h}}^{(n)}, n=1,2, \ldots$ satisfy the compatibility condition (3.2) if and only if for any $x \in V$ the following recursive equation 
holds:

$$
\left\{\begin{array}{c}
h_{x, 1}=\log \frac{\theta_{1} F_{1}\left(\theta_{x y}, \theta_{x z}, \kappa_{y z} ; \exp _{p}\left(h_{y}\right), \exp _{p}\left(h_{z}\right)\right)}{F_{2}\left(\theta_{x y}, \theta_{x z}, \kappa_{y z} ; \exp _{p}\left(h_{y}\right), \exp _{p}\left(h_{z}\right)\right)} \\
h_{x, 2}=\log \frac{\left.\theta_{1} F_{1}\left(\theta_{x y}, \theta_{x z}, \kappa_{y z} ; \exp _{p}\left(h_{y}\right)^{t}\right), \exp _{p}\left(\left(h_{z}\right)^{t}\right)\right)}{F_{2}\left(\theta_{x y}, \theta_{x z}, \kappa_{y z} ; \exp _{p}\left(\left(h_{y}\right)^{t}\right), \exp _{p}\left(\left(h_{z}\right)^{t}\right)\right)}
\end{array}\right.
$$

here $<y, x, z>$ ternary vertices, $\theta_{x y}=\exp _{p}\left\{J_{x y}\right\}, \kappa_{x y}=\exp _{p}\left\{K_{x y}\right\}$, $\theta_{1}=\exp _{p}(H)$ and for given vector $h=\left(h_{1}, h_{2}\right)$ by $\exp _{p}(h)$ and $h^{t}$ we have denoted the vectors $\left(\exp _{p}\left(h_{1}\right), \exp _{p}\left(h_{2}\right)\right)$ and $\left(h_{2}, h_{1}\right)$ respectively, and $F_{i}$ : $\mathbb{Q}_{p}{ }^{3} \times \mathbb{Q}_{p}{ }^{4} \rightarrow \mathbb{Q}_{p},(i=1,2)$ functions are defined by

$$
\left\{\begin{aligned}
F_{1}\left(\alpha_{1}, \alpha_{2}, \beta ; h, r\right)= & \alpha_{1} \alpha_{2} \beta h_{1} h_{2} r_{1} r_{2}+\alpha_{1} h_{1} h_{2}\left(r_{1}+r_{2}\right)+\alpha_{2} r_{1} r_{2}\left(h_{1}+h_{2}\right) \\
& +\beta\left(h_{1} r_{1}+h_{2} r_{2}\right)+h_{1} r_{2}+h_{2} r_{1} \\
F_{2}\left(\alpha_{1}, \alpha_{2}, \beta ; h, r\right)= & \beta h_{1} h_{2} r_{1} r_{2}+\alpha_{1} h_{1} r_{1} r_{2}+\alpha_{2} h_{1} h_{2} r_{1}+h_{2} r_{1} r_{2}+h_{1} h_{2} r_{2} \\
& +\alpha_{1} h_{1} r_{2}+\alpha_{2} h_{2} r_{1}+\beta h_{2} r_{2}+\alpha_{1} \alpha_{2} \beta h_{1} r_{1}
\end{aligned}\right.
$$

where $h=\left(h_{1}, h_{2}\right), r=\left(r_{1}, r_{2}\right)$.

Consequently, the problem of describing of $\mathcal{S}$ is reduced to the finding of solutions of the functional equation (3.3).

Write

$$
\Sigma=\left\{\mathbf{h}=\left(h_{x} \in \mathbb{Q}_{p}^{2}, x \in V\right): h_{x} \text { satisfies the equation (3.3) }\right\} .
$$

To prove the existence of phase transition it suffices to show that there are two different functions in $\Sigma$. The description of arbitrary elements of the set $\Sigma$ is a complicated problem.

Therefore, assume that $J_{x y}=J, K_{x y}=K$ and $H=0$. In this paper we restrict ourselves to the description of translation - invariant elements of $\Sigma$, i.e. in which $h_{x}=h$ is independent on $x$.

Let $h_{x}=h=\left(h_{1}, h_{2}\right)$ for all $x \in V$. Then using (3.4) we can reduce (3.3) to the following form

$$
\left\{\begin{array}{l}
u_{1}=\frac{\theta^{2} \kappa u_{1}^{2} u_{2}^{2}+2 \theta\left(u_{1}^{2} u_{2}+u_{1} u_{2}^{2}\right)+\kappa\left(u_{1}^{2}+u_{2}^{2}\right)+2 u_{1} u_{2}}{\kappa u_{1}^{2} u_{2}^{2}+2 \theta u_{1}^{2} u_{2}+2 u_{1} u_{2}^{2}+2 \theta u_{1} u_{2}+\kappa u_{2}^{2}+\theta^{2} \kappa u_{1}^{2}} \\
u_{2}=\frac{\theta^{2} \kappa u_{1}^{2} u_{2}^{2}+2 \theta\left(u_{1}^{2} u_{2}+u_{1} u_{2}^{2}\right)+\kappa\left(u_{1}^{2}+u_{2}^{2}\right)+2 u_{1} u_{2}}{\kappa u_{1}^{2} u_{2}^{2}+2 \theta u_{1} u_{2}^{2}+2 u_{1}^{2} u_{2}+2 \theta u_{1} u_{2}+\kappa u_{1}^{2}+\theta^{2} \kappa u_{2}^{2}}
\end{array}\right.
$$

here $u_{1}=\exp _{p}\left(h_{1}\right), u_{2}=\exp _{p}\left(h_{2}\right)$ and $\theta=\exp _{p}(J), \kappa=\exp _{p}(K)$.

From (3.5) it is easily seen that the lines $u_{1}=u_{2}, u_{1}=1$ and $u_{2}=1$, are invariant for the equation. Therefore, it is enough to consider the equation 
on the line $u_{2}=1$, since other cases can be reduced to this case. So, we rewrite (3.5) as follows

$$
u=\frac{\left(\theta^{2} \kappa+2 \theta+\kappa\right) u^{2}+2(\theta+1) u+\kappa}{2(\kappa+1) u^{2}+4 \theta u+\theta^{2} \kappa}
$$

It is evident that $u=1$ is a solution of (3.6), but to exist a phase transition we are interested for other solutions one. After some simple algebra we find the following equation

$$
2(\kappa+1) u^{2}+\left(2+2 \theta-\kappa-\theta^{2} \kappa\right) u-\kappa=0 .
$$

We have to find a solution of (3.7) such that $|u-1|_{p}<p^{-1 /(p-1)}$.

Rewriting (3.7) as follows

$$
2(\kappa+1)\left(u^{2}-1\right)+\left(2+2 \theta-\kappa-\theta^{2} \kappa\right)(u-1)+2 \theta-\theta^{2} \kappa+4=0
$$

we infer that if $\left|2 \theta-\theta^{2} \kappa+4\right|_{p}=1$ then (3.7) does not have a needed solution.

Therefore, we should require that $\left|2 \theta-\theta^{2} \kappa+4\right| \leq \frac{1}{p}$. We know (see (2.1)) that from the properties of the exponential function the parameters $\theta$ and $\kappa$ satisfy the following inequalities

$$
|\theta-1|_{p} \leq \frac{1}{p}, \quad|\kappa-1|_{p} \leq \frac{1}{p} .
$$

Using these inequalities we derive that the inequality $\left|2 \theta-\theta^{2} \kappa+4\right| \leq \frac{1}{p}$ is valid if and only if $p=3$. Therefore, let us assume that $p=3$. Denote

$$
P(x)=2(\kappa+1) x^{2}+\left(2+2 \theta-\kappa-\theta^{2} \kappa\right) x-\kappa .
$$

For $P(x)$ we have $P(1)=2 \theta-\theta^{2} \kappa+4, P^{\prime}(1)=6+2 \theta-\theta^{2} \kappa+3 \kappa$, hence by means of (3.8) one gets

$$
|P(1)|_{3} \leq \frac{1}{3}, \quad\left|P^{\prime}(1)\right|_{3}=1 .
$$

So according to the Hensel's lemma (see [13]) there is a solution $U \in \mathbb{Q}_{3}$ of the equation $P(x)=0$ such that $|U-1|_{3} \leq \frac{1}{3}$. Consequently, for the model under consideration there is a phase transition at $p=3$ for every $J, K$ such that $0<|J|_{3} \leq \frac{1}{3}, 0<|K|_{3} \leq \frac{1}{3}$.

\section{THE UnIQUenEsS OF THE GibBS MEASURE}

From the previous section we infer that if $p \neq 3$ then the equation (3.6) has a unique solution. Note that this solution corresponds to the case $h_{x}=$ $h$. Therefore, in general, does there exist a phase transition in this case or not? In this section we are going to answer this question. Here we will consider two cases. 
4.1. Non-homogeneous case. Let us assume that $H=0$ and (2.5) be satisfied. Then it is not hard to check that $\mathbf{h}=\left(h_{x}=0, x \in V\right)$ is a solution for (3.3). We will to prove that any other solution of (3.3) coincides with this one. To show it we have to estimate the value $\left\|h_{x}\right\|_{p}$. Denote $u_{x, i}=\exp _{p}\left(h_{x, i}\right), i=1,2$. Then from (2.1) we find

$$
\left|h_{x, i}\right|_{p}=\left|u_{x, i}-1\right|_{p}, \quad i=1,2 \text {. }
$$

hence we have

$$
\begin{aligned}
\left|u_{x, 1}-1\right|_{p}= & \left|\frac{F_{1}\left(\theta_{x y}, \theta_{x z}, \kappa_{y z} ; u_{y}, u_{z}\right)-F_{2}\left(\theta_{x y}, \theta_{x z}, \kappa_{y z} ; u_{y}, u_{z}\right)}{F_{2}\left(\theta_{x y}, \theta_{x z}, \kappa_{y z} ; u_{y}, u_{z}\right)}\right|_{p} \\
= & \mid \kappa_{y z} u_{y, 1} u_{z, 1}\left(\theta_{x y} \theta_{x z}-1\right)\left(u_{y, 2} u_{z, 2}-1\right)+u_{y, 1} u_{z, 2}\left(\theta_{x y}-1\right)\left(u_{y, 2}-1\right) \\
& +u_{y, 2} u_{z, 1}\left(\theta_{x z}-1\right)\left(u_{z, 1}-1\right)+\left.u_{y, 1} u_{z, 1}\left(\theta_{x y}-\theta_{x z}\right)\left(u_{y, 2}-u_{z, 2}\right)\right|_{p} \\
\leq & \frac{1}{p} \max \left\{\left|u_{y, 2} u_{z, 2}-1\right|_{p},\left|u_{y, 2}-1\right|_{p},\left|u_{z, 1}-1\right|_{p},\left|u_{y, 2}-u_{z, 2}\right|_{p}\right\} \\
= & \frac{1}{p} \max \left\{\left|h_{y, 2}+h_{z, 2}\right|_{p},\left|h_{y, 2}\right|_{p},\left|h_{z, 1}\right|_{p},\left|h_{y, 2}-h_{z, 2}\right|_{p}\right\} \\
\text { (4.2) } & \frac{1}{p} \max \left\{\left\|h_{y}\right\|_{p},\left\|h_{z}\right\|_{p}\right\}
\end{aligned}
$$

here we again used (2.1) and

$$
\left|F_{2}\left(\theta_{x y}, \theta_{x z}, \kappa_{y z} ; u_{y}, u_{z}\right)\right|_{p}=1
$$

which is valid if $p \neq 3$.

Analogously reasoning we derive

$$
\left|u_{x, 2}-1\right|_{p} \leq \frac{1}{p} \max \left\{\left\|h_{y}\right\|_{p},\left\|h_{z}\right\|_{p}\right\} .
$$

The equality (4.1) with (4.2),(4.3) implies that

$$
\left\|h_{x}\right\|_{p} \leq \frac{1}{p} \max \left\{\left\|h_{y}\right\|_{p},\left\|h_{z}\right\|_{p}\right\} .
$$

Take an arbitrary $\varepsilon>0$. Let $n_{0} \in \mathbb{N}$ be such that $\frac{1}{p^{n} 0}<\varepsilon$. Now iterating (4.4) $n_{0}$ times one gets

$$
\left\|h_{x}\right\|_{p} \leq \frac{1}{p^{n_{0}}}<\varepsilon
$$

this means that $h_{x}=0$ for all $x \in V$. Thus, the $p$-adic Gibbs measure is unique. 
4.2. Homogeneous case. In this subsection we will assume that $J_{x y}=J$, $K_{x y}=K$ and $H \neq 0$. Let us suppose that $h_{x}=h=\left(h_{1}, h_{2}\right)$ for all $x \in V$. In this case one easily sees that (3.3) invariant with respect to $u_{1}=u_{2}$. Therefore, we are looking for a solution of (3.3) of the form $\left(h_{1}, h_{1}\right)$. Then it can be rewritten as follows

$$
u=\theta_{1} \frac{\theta^{2} \kappa u^{2}+4 \theta u+2(\kappa+1)}{\kappa u^{2}+2(\theta+1) u+\theta^{2} \kappa+2 \theta+\kappa}, \quad u=\exp _{p}\left(h_{1}\right) .
$$

Denote

$$
f(x)=\theta_{1} \frac{\theta^{2} \kappa x^{2}+4 \theta x+2(\kappa+1)}{\kappa x^{2}+2(\theta+1) x+\theta^{2} \kappa+2 \theta+\kappa} .
$$

Let us show that $f\left(B\left(1, p^{-1 /(p-1)}\right)\right) \subset B\left(1, p^{-1 /(p-1)}\right)$. Indeed, let $\mid x-$ $\left.1\right|_{p}<p^{-1 /(p-1)}$, then

$$
\begin{aligned}
|f(x)-1|_{p}= & \left|\frac{\left(\theta^{2} \theta_{1} \kappa-\kappa\right) x^{2}+\left(4 \theta \theta_{1}-2 \theta-2\right) x+2(\kappa+1) \theta_{1}-\theta^{2} \kappa-2 \theta-\kappa}{\kappa x^{2}+2(\theta+1) x+\theta^{2} \kappa+2 \theta+\kappa}\right|_{p} \\
\leq & \max \left\{\left|\left(\theta^{2} \kappa \theta_{1}-\kappa\right)\left(x^{2}-1\right)\right|_{p},\left|\left(4 \theta \theta_{1}-2 \theta-2\right)(x-1)\right|_{p},\right. \\
& \left.\left|\left(\theta^{2} \kappa+4 \theta+2(\kappa+1)\right)\left(\theta_{1}-1\right)\right|_{p}\right\} \\
\leq & \max \left\{|(x-1)|_{p},\left.\left(\theta_{1}-1\right)\right|_{p}\right\}<p^{-1 /(p-1)}
\end{aligned}
$$

here we have used the equality $\left|\kappa x^{2}+2(\theta+1) x+\theta^{2} \kappa+2 \theta+\kappa\right|_{p}=1$ which is valid if $p \neq 3$.

Now after some algebra we derive

$$
\begin{aligned}
|f(x)-f(y)|_{p}= & \mid 2 \theta \kappa x y\left(2-\theta-\theta^{2}\right) \\
& +\kappa(x+y)\left(2(\kappa+1)-\theta^{2}\left(\theta^{2} \kappa+2 \theta+\kappa\right)\right) \\
& +\left.4\left((\theta+1)(\kappa+1)-\theta^{2} \kappa-2 \theta-\kappa\right)\right|_{p}|x-y|_{p} \\
\leq & \max \left\{\left|2-\theta-\theta^{2}\right|,\right. \\
& \left|2(\kappa+1)-\theta^{2}\left(\theta^{2} \kappa+2 \theta+\kappa\right)\right|_{p}, \\
& \left.\left|(\theta+1)(\kappa+1)-\theta^{2} \kappa-2 \theta-\kappa\right|_{p}\right\}|x-y|_{p} .
\end{aligned}
$$


Using (3.8) one can be shown that

$$
\left\{\begin{array}{l}
\left|2-\theta-\theta^{2}\right| \leq \frac{1}{p} \\
\left|2(\kappa+1)-\theta^{2}\left(\theta^{2} \kappa+2 \theta+\kappa\right)\right|_{p} \leq \frac{1}{p}, \\
\left|(\theta+1)(\kappa+1)-\theta^{2} \kappa-2 \theta-\kappa\right|_{p} \leq \frac{1}{p}
\end{array}\right.
$$

The last inequalities with (4.7) imply that

$$
|f(x)-f(y)|_{p} \leq \frac{1}{p}|x-y|_{p}
$$

Thus the inequality (4.8) yields that $f$ is a contraction of $B\left(1, p^{-1 /(p-1)}\right)$, hence $f$ has a unique fixed point $\zeta \in B\left(1, p^{-1 /(p-1)}\right)$. Let $\xi=\log _{p} \zeta$ and $\bar{\xi}=(\xi, \xi)$. Then using the same argument as (4.2) we may obtain

$$
\left\|h_{x}-\bar{\xi}\right\|_{p} \leq \frac{1}{p} \max \left\{\left\|h_{y}-\bar{\xi}\right\|_{p},\left\|h_{z}-\bar{\xi}\right\|_{p}\right\} .
$$

Now using the argument of the previous subsection we get that $h_{x}=\bar{\xi}$ for all $x \in V$.

Thus, the $p$-adic Gibbs measure is unique.

\section{CONCLUSiOnS}

In the paper we have considered three state $p$-adic Potts model with competing interactions on a Cayley tree order two. We reduced a problem of describing of the $p$-adic Gibbs measures to the solution of certain recursive equation, and using it we proved that a phase transition occurs if and only if $p=3$ for any value (non zero) of interactions. If $p \neq 3$ we showed that there is only a unique Gibbs measure for the inhomogeneous Potts model with zero external filed, as well as we established that result for homogeneous model but with non-zero external filed. From these results we conclude that a phase transition depends only on a value of $p$. These results totaly different from the real case, since in this setting there is a phase transition on some constraints for the interaction parameters (see [3],[18]). When a $p$ adic Gibbs measure is unique, then by means of a method of paper [16] one can be shown that the measure is bounded as well. The results concerning the uniqueness of the Gibbs measures extend results obtained in $[16,17]$. We hope our results will force to study certain limit theorems for such kind of measures, since they naturally appear from some Hamiltonian systems, and on the other hand, these measures enable a Markov property. We also hope that these investigations give some opportunity to study Hamiltonian systems over networks in a $p$-adic setting [2]. 


\section{ACKNOWLEDGMENTS}

The authors would like express their gratitude the organizers of the conference on $p$-adic Mathematical Physics for an invitation. F.M. thanks the FCT (Portugal) grant SFRH/BPD/17419/2004. F.M. and U.R thank also for grant $\Phi-2.1 .56$ of CST of Uzbekistan. U.R. acknowledges prof. M.Cassandro for an invitation to "La Sapienza" University and NATO Reintegration Grant FEL.RIG.980771.

\section{REFERENCES}

[1] I.Ya.Aref'eva, B.Dragovich, P.H.Frampton and I.V.Volovich, Int. J. Mod. Phys. A. 6, 4341-4358 (1991).

[2] S.N. Dorogovtsev, A.V. Goltsev and J.F.F. Mendes, Phys. Rev. E 67, 026123 (2003).

[3] N.N. Ganikhodjaev and U.A. Rozikov, Osaka Jour. Math. 37, 373-383 (2000).

[4] H.O. Georgii, Gibbs measures and phase transitions, Walter de Gruyter, Berlin, 1988.

[5] S.Katsura and M. Takizawa, Progr. Theor. Phys. 51, 82-98 (1974).

[6] A.Yu.Khrennikov, J.Math.Phys. 32, 932-936 (1991).

[7] A.Yu.Khrennikov, p-adic Valued Distributions in Mathematical Physics. Kluwer Academic Publisher, Dordrecht, 1994.

[8] A.Yu.Khrennikov, $p$-adic probability distribution of hidden variables, Physica A, 215 (1995), 577-587.

[9] A.Yu.Khrennikov, Indag. Mathem. N.S. 7, 311-330 (1996).

[10] A.Yu.Khrennikov, Statis.Probab. Lett. 51, 269-276 (2001).

[11] A.Yu.Khrennikov and S.Ludkovsky, Markov Process. Related Fields 9, 131-162 (2003).

[12] A.Yu.Khrennikov and S. Yamada, Theor. Probab. Appl. 49, 65-76 (2005).

[13] N.Koblitz, p-adic numbers, p-adic analysis and zeta-function, Berlin, Springer, 1977.

[14] E.Marinary and G.Parisi, Phys.Lett. 203B, 52-56 (1988).

[15] M.Mariz, C.Tsalis and A.L.Albuquerque, Jour. of Stat. Phys. 40, 577-592 (1985).

[16] F.M. Mukhamedov and U.A. Rozikov, Indag. Mathem. N.S. 15, 85-100 (2004).

[17] F.M. Mukhamedov and U.A. Rozikov, Infin. Dimens. Anal. Quantum Probab. Relat. Top. 8, 277-290 (2004).

[18] F.M. Mukhamedov and U.A. Rozikov, J. Stat. Phys. 114 825-848 (2004); 119, 427446 (2005).

[19] D.Ruelle, Statistical Mechanics: Rigorus Results, Benjamin, 1969.

[20] C.R. da Silca and S. Coutinho, Phys. Review B,34, 7975-7985 (1986).

[21] J.Vannimenus, Z.Phys. B 43, 141 (1981).

[22] V.S.Vladimirov, I.V.Volovich and E.I.Zelenov, p-adic Analysis and Mathematical Physics, Singapour, World Scientific, 1994. 
F.M. Mukhamedov, Departamento de Fisica, Universidade de Aveiro, CAmpus Universitrio de SAntiago, 3810-193 Aveiro, Portugal

E-mail address: far75meyandex.ru, farruhefis.ua.pt

U.A. Rozikov, Institute of Mathematics, 29, F.Hodjaev str., Tashkent, 700125, UZBEKISTAN

E-mail address: rozikovueyandex.ru

J.F.F. Mendes, Departamento de Fisica, Universidade de Aveiro, Campus Universitrio de SAntiago, 3810-193 Aveiro, Portugal

E-mail address: jfmendesefis.ua.pt 